plasma cells in the GCs (Figure 2B). These two events may synergize and lead to higher levels of SIV-specific antibodies. A detailed analysis of the cellular components expressing PD-1 and its ligands needs to be performed in the GCs of SIV-infected animals to clarify the cellular components involved in PD-1 blockade.

\section{Concluding remarks}

There is a need to develop innovative therapeutic strategies to ameliorate responses to common vaccination antigens in HIV-1infected children and adults $(3,6)$, since limited access to ART and fast progression to AIDS upon HIV-1 infection in developing countries impairs responses to vaccination with bacterial and viral antigens in HIV-1-infected subjects. The present study from Titanji and colleagues (3) paves the way for a new treatment intervention, based on PD-1 blockade, that aims to ameliorate $\mathrm{B}$ cell responses. By reducing $\mathrm{T}$ cell exhaustion (17) and by improving $B$ cell responses (3), this PD-1-based approach may provide a platform to accelerate immune reconstitution during HIV-1 infection.

\section{Acknowledgments}

The author's work is supported through grants from the Swedish MRC, the Swedish International Development Agency
(SIDA-SAREC), the Karolinska Institutet, the Fp6 EU Europrise network of excellence, the Fp7 EU NGIN Collaborative project Health-F3-2007-201433, and the regional agreement on medical training and clinical research (ALF) between Stockholm County Council and Karolinska Institutet.

Address correspondence to: Francesca Chiodi, Department of Microbiology, Tumor and Cell Biology, Karolinska Institutet, Nobels väg 16, S-17177 Stockholm, Sweden. Phone: 46.8.52486315; Fax: 46.8.330498; E-mail: francesca.chiodi@ki.se.

1. INSIGHT-ESPRIT Study Group, et al. Interleukin-2 therapy in patients with HIV infection. $N$ Engl J Med. 2009;361(16):1548-1559.

2. De Milito A, et al. Mechanisms of hypergammaglobulinemia and impaired antigen-specific humoral immunity in HIV-1 infection. Blood. 2004; 103(6):2180-2186

3. Titanji K, et al. Acute depletion of activated memory $\mathrm{B}$ cells involves the PD-1 pathway in rapidly progressing SIV-infected macaques. J Clin Invest. 2010; 120(11):3878-3890.

4. Moir S, Fauci AS. B cells in HIV infection and disease. Nat Rev Immunol. 2009;9(4):235-245.

5. Cagigi A, Nilsson A, Pensieroso S, Chiodi F. Dysfunctional B-cell responses during HIV-1 infection: implication for influenza vaccination and highly active antiretroviral therapy. Lancet Infect Dis. 2010;10(7):499-503.

6. Titanji K, et al. Primary HIV-1 infection sets the stage for important B lymphocyte dysfunctions. AIDS. 2005;19(17):1947-1955.
7. Titanji K, et al. Loss of memory B cells impairs maintenance of long-term serologic memory during HIV-1 infection. Blood. 2006;108(5):1580-1587.

8. Pensieroso $\mathrm{S}$, et al. Timing of HAART defines the integrity of memory B cells and the longevity of humoral responses in HIV-1 verticallyinfected children. Proc Natl Acad Sci U S A. 2009; 106(19):7939-7944.

9. Peruchon S, et al. Tissue-specific B-cell dysfunction and generalized memory B-cell loss during acute SIV infection. PLoS One. 2009;4(6):e5966.

10. Cagigi A, et al. Altered expression of the receptor-ligand pair CXCR5/CXCL13 in B cells during chronic HIV-1 infection. Blood. 2008; 112(12):4401-4410.

11. Okazaki T, Honjo T. The PD-1-PD-L pathway in immunological tolerance. Trends Immunol. 2006; 27(4):195-201.

12. Okazaki T, Maeda A, Nishimura H, Kurosaki T, Honjo T. PD-1 immunoreceptor inhibits B cell receptor-mediated signaling by recruiting src homology 2-domain-containing tyrosine phosphatase 2 to phosphotyrosine. Proc Natl Acad SciU S A. 2001;98(24):13866-13871.

13. Okazaki T, et al. Autoantibodies against cardiac troponin I are responsible for dilated cardiomyopathy in PD-1-deficient mice. Nat Med. 2003;9(12):1477-1483.

14. Good-Jacobson KL, Szumilas CG, Chen L, Sharpe $\mathrm{AH}$, Tomayko MM, Shlomchik MJ. PD-1 regulates germinal center $\mathrm{B}$ cell survival and the formation and affinity of long-lived plasma cells. Nat Immunol. 2010;11(6):535-542.

15. Nutt SL, Tarlinton DM. Give and take in the germinal center. Nat Immunol. 2010;11(6):464-466.

16. Trabattoni $\mathrm{D}$, et al. B7-H1 is up-regulated in HIV infection and is a novel surrogate marker of disease progression. Blood. 2003;101(7):2514-2520.

17. Velu V, et al. Enhancing SIV-specific immunity in vivo by PD-1 blockade. Nature. 2009; 458(7235):206-210.

\title{
Pharmacologic eigenvalues: beating the rap on bone marrow failure
}

\author{
Stephen G. Emerson ${ }^{1,2}$ and Russell W. Garrett ${ }^{1}$ \\ ${ }^{1}$ Department of Biology, Haverford College, Haverford, Pennsylvania, USA. ${ }^{2}$ Department of Medicine and Abramson Cancer Center, \\ University of Pennsylvania, Philadelphia, Pennsylvania, USA.
}

\begin{abstract}
Patients suffering from sustained acute or chronic illness often have decreased white blood cell and platelet counts as well as anemia, and bone marrow studies routinely show only decreased numbers of blood precursor cells. While much has been recently learned about the cause of isolated anemia, the pathogenesis of true bone marrow failure (i.e., low bone marrow cellularity and low counts in multiple blood lineages) has remained elusive. In this issue of the JCI, Chen et al. present evidence that overactivation of mammalian target of rapamycin signaling in HSCs is found in two mouse models of bone marrow failure, and they show that treatment with rapamycin significantly normalizes the low blood counts.
\end{abstract}

Conflict of interest: The authors have declared that no conflict of interest exists.

Citation for this article: J Clin Invest. doi:10.1172/ JCI45060.
The recent explosions in genomics and systems biology highlight the usefulness of mathematic techniques, such as differential equations and linear algebra, for the manip- ulation and analysis of large data sets. One powerful mathematical concept for simplifying and understanding such complex systems is the eigenvalue, a dominant vector within a complex linear system, around which other vectors rotate and relate. The effects of an eigenvalue explain and dominate those of others in the system.

The concept of the eigenvalue has important implications beyond mathematical models. It is especially important in experimental biology and clinical medicine, as investigators search for single molecules or pathways whose effects dominate complex reactions within a cell. Even if we do not always have at hand detailed mathematical models and thus 
cannot always strictly identify eigenvalues a priori, specific pharmacologic interventions can sometimes reveal them experimentally, thereby simplifying an otherwise bewildering network of interacting pathways.

In this issue of the JCI, Chen et al. (1), provide us with an intriguing example of a biological eigenvalue unveiled by pharmacologic manipulation. They present compelling evidence linking acute and chronic activation of the innate immune system to suppression of HSC differentiation. They go on to identify the mammalian target of rapamycin (mTOR) pathway as a dominant signal transduction pathway mediating this effect. These results link chronic inflammation with stem cell aging and, furthermore, make the powerful suggestion that treatment with the mTOR inhibitor rapamycin might mitigate these effects.

\section{Hematopoiesis is regulated by the immune system: growth factors and cytokines}

Ever since MHC class II antigens were found to be expressed on early myeloid progenitor cells as well as traditional antigen-presenting cells (2), the hematopoietic system has been appreciated as a potential regulator and target of adaptive immune activity. Indeed, T cells activated via engagement of their TCRs and costimulatory molecules by activating cells (perhaps including hematopoietic progenitors themselves) promote myeloid differentiation through the secretion of GM-CSF and IL-3, resulting in increased neutrophil and red blood cell production from HSCs, both under basal conditions and after stem cell transplantation $(3,4)$.

In this issue of the JCI, Chen et al. (1) show that extremely high levels of activation of the innate immune system, either through the global inactivation of the gene encoding forkhead box P3 (FoxP3) in scurfy mice or by injection of LPS, severely abrogate HSC differentiation, resulting in profound loss of $\mathrm{B}$ lymphoid, neutrophilic, and erythropoietic lineages. These effects could be largely prevented through inhibition of TNF- $\alpha$, IL- 6 , and CCL2 (but not IFN- $\gamma$ ). Chen and colleagues went on to show that both TNF- $\alpha$ and IL- 6 induce phosphorylation of the mTOR target ribosomal protein S6 kinase (S6K) in HSCs (1). Perhaps most remarkably, inhibition of the mTOR pathway with rapamycin largely ameliorated these suppressive effects in both mouse models. Clearly, innate immune activation involves the action of multiple soluble cytokines and insoluble ligand-receptor interactions, which in turn trigger multiple cellular pathways, of which mTOR is only one. However, mTOR in HSCs must act as a "biological eigenvalue," coordinating most of the inhibitory effects in HSCs of this complex set of intracellular responses to external signals of innate immune activation. Thus, while TNF- $\alpha$, IL-6, and CCL2 activate multiple signal transduction pathways within target cells, not the mTOR pathway alone, the striking effects of rapamycin demonstrate that among these pathways, mTOR plays an integrating, dominant role, at least within early hematopoietic cells.

\section{High levels of TNF- $\alpha$ cause overt HSC damage}

How mTOR activation prevents HSCs from proliferating and differentiating to produce mature blood cells is less clear but appears likely to be the result of direct stem cell toxicity via unclear mechanisms. Chen et al. found that the number of bone marrow cells expressing the cell surface proteins that typically appear on HSCs actually rose slightly, even while hematopoiesis declined, but many of these HSCs were dead or dying (1). Thus, the apparent increase in cells bearing HSC markers is likely to be an artifact of the stress induced by the acute toxicity of high levels of activation of the innate immune system, which includes high levels of circulating cytokines.

Direct damage of HSCs might underlie recent findings from other laboratories that, like TNF- $\alpha$, chronic IFN stimulation causes an apparent increase in cells detected as expressing markers of HSCs but a decrease in HSC function. This artifact is most clear in the case of IFN-induced increases in the number of Sca- $1^{+} \mathrm{ckit}^{-} \mathrm{lin}^{-}$ cells (the traditional cell surface signature of HSCs) that were determined to occur not because HSCs proliferate but, instead, because cell surface expression of Sca-1 was directly induced on Sca-1- non-HSCs $(5,6)$. Cell surface Sca-1 has also been demonstrated to be induced on previously Sca- $1^{-}$ hematopoietic cells by TNF- $\alpha$ (7), and this might at least partially explain the findings of Chen et al. (1). Whether alterations in cell surface proteins other than Sca-1 can explain the apparent lack of increase in hematopoiesis despite increased numbers of cells apparently marking as HSCs in the paper by Chen et al. (1) is not clear, but it is clearly possible. In any case, the results of Chen and colleagues make it clear that direct cell damage can readily be detected on early hematopoietic cells (1), which include both HSCs and slightly more dif- ferentiated non-HSCs. Most importantly, no study has yet shown an increase in the number of functional, transplantable HSCs after exposure to cytokine mediators of inflammation, including IFN, TNF- $\alpha$, and IL-6, i.e., numbers of cells with a particular cell surface phenotype do not equal numbers of functional stem cells. Limiting dilution transplantation experiments to quantitatively probe this possibility would be very interesting. But whether cells marking as HSCs increase in number or not after extremely high levels of activation of the innate immune system, the important point is that from a functional point of view, they are not able to fend off the powerful effects of the innate immune system on HSC integrity and differentiation capacity, irrespective of stem cell cycling per se.

\section{mTOR, rapamycin, and the complications of innate immune activation}

Whether or not it is true that HSC numbers rise, the significance of functional hematopoietic suppression by mediators of inflammation is real enough. The findings of Chen et al. (1) will clearly intrigue every clinician who has cared for severely ill patients, often in intensive care units, whose pancytopenia defies both explanation and treatment. Perhaps, as suggested by Chen et al. (8), mTOR inhibition acts to prevent premature stem cell aging and exhaustion induced under the veil of innate immune activation. The possibility that short-term rapamycin treatment could ameliorate this and perhaps other manifestations of pathologic cytokine activation should inspire careful clinical trials in the future. Such trials will exemplify the clinical investigator's understanding and application of eigenvalues, both mathematical and biological, to the untangling of the complex web of the cell for the understanding and treatment of disease.

Address correspondence to: Stephen G. Emerson, Sharpless Hall, Haverford College, 370 Lancaster Avenue, Haverford, Pennsylvania 19041, USA. Phone: 610.896.1021; Fax:610.896.2402; E-mail: semerson@ haverford.edu.

Russell W. Garrett is deceased.

1. Chen C, Liu Y, Liu Y, Zheng P. Mammalian target of rapamycin activation underlies HSC defects in autoimmune disease and inflammation in mice. J Clin Invest. 2010;120(11):4091-4101.

2. Winchester RJ, Ross GD, Jarowski CI, Wang CY, Halper J, Broxmeyer HE. Expression of Ia-like anti- 
gen molecules on human granulocytes during early phases of differentiation. Proc Natl Acad Sci U S A. 1977;74(9):4012-4016.

3. Guba SC, Stella G, Turka LA, June CH, Thompson $\mathrm{CB}$, Emerson SG. Regulation of interleukin 3 gene induction in normal human $\mathrm{T}$ cells. J Clin Invest. 1989;84(6):1701-1706

4. Hexner EO, et al. Umbilical cord blood xenografts in immunodeficient mice reveal that $\mathrm{T}$ cells enhance hematopoietic engraftment beyond overcoming immune barriers by stimulating stem cell differentiation. Biol Blood Marrow Transplant. 2007;13(10):1135-1144.

5. Essers MA, et al. IFNalpha activates dormant haematopoietic stem cells in vivo. Nature. 2009; 458(7240):904-908

6. Baldridge MT, King KY, Boles NC, Weksberg DC, Goodell MA. Quiescent haematopoietic stem cells are activated by IFN-gamma in response to chronic infection. Nature. 2010;465(7299):793-797.
7. Luna G, Paez J, Cardier JE. Expression of the hematopoietic stem cell antigen Sca-1 (LY-6A/E) in liver sinusoidal endothelial cells: possible function of Sca-1 in endothelial cells. Stem Cells Dev. 2004;13(5):528-535.

8. Chen C, Liu Y, Liu R, Ikenoue T, Guan KL, Zheng P. TSC-mTOR maintains quiescence and function of hematopoietic stem cells by repressing mitochondrial biogenesis and reactive oxygen species. J Exp Med. 2008;205(10):2397-2408.

\title{
The cellular response to hypoxia: tuning the system with microRNAs
}

\author{
Joseph Loscalzo \\ Department of Medicine, Brigham and Women's Hospital, Harvard Medical School, Boston, Massachusetts, USA.
}

\begin{abstract}
Adaptation to hypoxia is an essential cellular response controlled by the oxygen-sensitive master transcription factor hypoxia-inducible factor 1 (HIF-1). HIF-1 expression is also controlled by specific microRNAs and, in turn, controls the expression of other microRNAs, which fine-tune adaptation to low oxygen tension. In this issue of the JCI, Ghosh and colleagues identify a unique microRNA in hypoxic endothelial cells, miR424, that promotes HIF-1 stabilization and angiogenesis. The actions of this microRNA are considered in the context of the complex interactions that act to ensure optimal endothelial adaptation to this critical environmental condition.
\end{abstract}

Adaptation to hypoxia is an essential homeostatic mechanism in mammalian cells. Hypoxia can occur not only as a global consequence of low atmospheric oxygen tension, but also locally at sites of inflammation, tissue ischemia and injury, and solid tumor growth. Fundamental mechanisms have, therefore, evolved by which cells adapt to hypoxic conditions and their potentially adverse consequences, both acutely and chronically, in health and disease.

Among the key responses to acute hypox$i a$ is the upregulation of hypoxia-inducible factors (HIFs), master transcription factors that induce the highly conserved expression of many genes (likely more than 100) and are responsible for the majority of the hypoxic program in metazoan cells (1). The prototypical HIF is a heterodimer of $\alpha$ and $\beta$ subunits. Under normoxic conditions, the $\alpha$ subunit is rapidly hydroxylated at specific prolyl residues by prolyl hydroxylase domain (PHD) proteins. Upon hydroxylation, the $\alpha$ subunit is recognized by the von HippelLindau protein and consequently undergoes rapid proteasomal degradation. Under

Conflict of interest: The author has declared that no conflict of interest exists.

Citation for this article: J Clin Invest. doi:10.1172/ JCI45105. hypoxic conditions, however, the $\alpha$ subunit does not undergo prolyl hydroxylation and, as a result, forms a stable complex with the $\beta$ subunit, which binds as a heterodimer to hypoxia response elements (HREs) in the promoter regions of hypoxia-sensitive genes to induce gene transcription. HIF-dependent transcriptional changes lead to a broad range of cellular adaptations, including metabolic, proliferative, apoptotic, and angiogenic. In higher metazoans, three different HIF- $\alpha$ proteins have been identified: HIF- $1 \alpha$ and HIF- $2 \alpha$ have some transcriptional targets in common and some that are unique to each subunit, while splice variants of HIF-3 $\alpha$ have dominant negative effects on HIFdependent gene transcription $(2,3)$.

\section{MicroRNAs and transcriptional tuning}

MicroRNA molecules (miRNAs) are essential, noncanonical RNA species of 18-23 nucleotides in length that regulate gene expression. There are believed to be more than 1,000 miRNA genes in the human genome, which are estimated to regulate more than onethird of all mRNA transcripts (4). As part of the RNA-induced silencing complex (RISC), miRNAs negatively regulate gene transcription by annealing to the $3^{\prime}$ untranslated region of specific mRNA targets to repress translation, enhance mRNA degradation, or both. In contrast to the promoter-based catalytic amplification of mRNA synthesis, miRNAs are viewed as weak modulators of the transcriptional response, fine-tuning gene expression largely by negative regulation in a process that requires stoichiometric binding to mRNA targets (5).

\section{Hypoxamirs: microRNAs induced by hypoxia}

Hypoxia has recently been shown to induce the expression of a number of miRNAs, which have been termed "hypoxamirs" (6). Owing to the central importance of HIFs to the hypoxic response, its potent regulation of many genes whose expression is regulated by oxygen tension, and the significant number of hypoxamirs, it is logical to explore the relationships among HIFs and hypoxamirs in order to understand better the complex regulation of the hypoxic program in metazoans.

From the perspective of the miRNA response to hypoxia, miRNAs can be viewed as comprising three groups (Table 1): first, those induced by HIFs under hypoxic conditions (HIF-dependent hypoxamirs); second, those induced by hypoxia that, in turn, affect expression of HIF; third, those whose expression is not dependent on hypoxia but that affect HIF expression. The number of hypoxamirs that have been identified in each of these groups is expanding rapidly, illustrating the extraordinary regulatory complexity of the hypoxic program and the importance of considering that program in a holistic, system-based context. In this issue of the JCI, Ghosh and colleagues (7) identify a new hypoxamir, miR424, that affects HIF expression. This report is unique for three reasons: it is the first demonstration of the 